\author{
P. Flachs $\cdot$ O. Horakova $\cdot$ P. Brauner $\cdot$ M. Rossmeisl · \\ P. Pecina $\cdot$ N. Franssen-van Hal $\cdot$ J. Ruzickova • \\ J. Sponarova $\cdot$ Z. Drahota $\cdot$ C. Vlcek $\cdot$ J. Keijer $\cdot$ \\ J. Houstek · J. Kopecky
}

\title{
Polyunsaturated fatty acids of marine origin upregulate mitochondrial biogenesis and induce $\beta$-oxidation in white fat
}

Received: 10 March 2005 / Accepted: 13 June 2005 / Published online: 5 October 2005

(C) Springer-Verlag 2005

\begin{abstract}
Aims/hypothesis: Intake of $n-3$ polyunsaturated fatty acids reduces adipose tissue mass, preferentially in the abdomen. The more pronounced effect of marine-derived eicosapentaenoic (EPA) and docosahexaenoic (DHA) acids on adiposity, compared with their precursor $\alpha$-linolenic acid, may be mediated by changes in gene expression and metabolism in white fat. Methods: The effects of EPA/ DHA concentrate (6\% EPA, 51\% DHA) admixed to form two types of high-fat diet were studied in C57BL/6J mice. Oligonucleotide microarrays, cDNA PCR subtraction and quantitative real-time RT-PCR were used to characterise gene expression. Mitochondrial proteins were quantified using immunoblots. Fatty acid oxidation and synthesis were measured in adipose tissue fragments. Results: Expression screens revealed upregulation of genes for mitochondrial proteins, predominantly in epididymal fat when EPA/DHA
\end{abstract}

Electronic Supplementary Material Supplementary material is available in the online version of this article at http://dx.doi. org/10.1007/s00125-005-1944-7

P. Flachs $\cdot$ O. Horakova $\cdot$ P. Brauner $\cdot$ M. Rossmeisl ·

J. Ruzickova $\cdot$ J. Sponarova $\cdot$ J. Kopecky $(\bowtie)$

Department of Adipose Tissue Biology, Institute of Physiology,

Academy of Sciences of the Czech Republic,

Videnska 1083

14220 Prague, Czech Republic

e-mail: kopecky@biomed.cas.cz

Tel.: +420-24106255

Fax: $+420-241062599$

P. Pecina $\cdot$ Z. Drahota $\cdot$ J. Houstek

Department of Bioenergetics,

Institute of Physiology,

Academy of Sciences,

Prague, Czech Republic

N. Franssen-van Hal · J. Keijer

RIKILT-Institute of Food Safety,

Wageningen, The Netherlands

C. Vlcek

Department of Genomics and Bioinformatics,

Institute of Molecular Genetics, Academy of Sciences,

Prague, Czech Republic concentrate was admixed to a semisynthetic high-fat diet rich in $\alpha$-linolenic acid. This was associated with a threefold stimulation of the expression of genes encoding regulatory factors for mitochondrial biogenesis and oxidative metabolism (peroxisome proliferator-activated receptor gamma coactivator 1 alpha [Ppargcla, also known as Pgcl $\alpha]$ and nuclear respiratory factor-1 [Nrfl] respectively). Expression of genes for carnitine palmitoyltransferase 1A and fatty acid oxidation was increased in epididymal but not subcutaneous fat. In the former depot, lipogenesis was depressed. Similar changes in adipose gene expression were detected after replacement of as little as $15 \%$ of lipids in the composite highfat diet with EPA/DHA concentrate, while the development of obesity was reduced. The expression of Ppargcla and $N r f 1$ was also stimulated by $n-3$ polyunsaturated fatty acids in 3T3-L1 cells. Conclusions/interpretation: The antiadipogenic effect of EPA/DHA may involve a metabolic switch in adipocytes that includes enhancement of $\beta$-oxidation and upregulation of mitochondrial biogenesis.

Keywords Adipose tissue - Carnitine palmitoyltransferase 1 - Fish oil · Flax-seed oil · Metabolism - Mitochondria · n-3 Polyunsaturated fatty acids - PGC-1 - PPARGC1A

Abbreviations ACOX1: acyl-Coenzyme A oxidase 1 . ALA: $\alpha$-linolenic acid - ATP5A1: subunit $\alpha$ of $F_{1}$-ATPase . AU: arbitrary units $\cdot \mathrm{cHF}$ diet: composite high-fat diet based on chow $\cdot$ cHF-F1 and sHFf-F2: high-fat diets with partial replacement of lipids by fish oil concentrate COX6: cytochrome c oxidase subunit VI - CPT1A: carnitine palmitoyltransferase 1A - DHA: docosahexaenoic acid - EPA: eicosapentaenoic acid - EPA/DHA concentrate: concentrate of $n-3$ polyunsaturated fatty acids from fish oil (6\% EPA and 51\% DHA) $\cdot$ ESM: Electronic Supplementary Material - MT-ATP6 and MT-ATP8: subunits 6 and 8 of mitochondrial ATPase $\cdot$ MT-CO1 and MT-CO3: mitochondrial cytochrome $c$ oxidase subunits I and III - MT-ND1: subunit 1 of mitochondrial NADH dehydrogenase (complex I) · MT-ND4: subunit 4 of mitochondrial NADH dehydrogenase (complex I) . NRF1: nuclear respiratory factor-1 PPAR: peroxisome 
proliferative activated receptor - PPAR GC1A: PPAR $\gamma$ coactivator- $1 \alpha \cdot$ PTP4A1: protein tyrosine phosphatase 4a1 - PUFA: polyunsaturated fatty acids - qRT-PCR: quantitative real-time RT-PCR - SCD1: stearoyl-CoA desaturase $1 \cdot$ SDHA: $70 \mathrm{kDa}$ protein subunit of mitochondrial complex II - sHFf diet: semisynthetic high-fat diet $\cdot$ UCP: uncoupling protein

\section{Introduction}

Type 2 diabetes exhibits a much stronger dependence on obesity than other health consequences of excessive accumulation of body fat. One of the major causes of obesity is high intake of lipids [1]. Studies in rodents [2-7] have demonstrated that saturated fats facilitate adipose tissue deposition more effectively than polyunsaturated fatty acids (PUFA). The long-chain $n$-3 PUFA eicosapentaenoic acid (EPA; 20:5 n-3) and docosahexaenoic acid (DHA, 22:6n-3), which are abundant in marine fish oil, are more potent in reducing adiposity than $n$-3 PUFA from plants, such as $\alpha$-linolenic acid (ALA, 18:3 $n$-3 [4, 8]). The intake of DHA appears to be particularly important $[5,8]$. In mammals, ALA is a precursor of EPA and DHA, but is rapidly oxidised in the organism and its conversion to EPA and DHA is quite inefficient $[6,9,10]$. Hence, dietary fish oil supplement is a more effective source of tissue DHA than ALA [6, 10]. Also in humans, dietary fish oil may decrease body fat content [11]. Furthermore, EPA and DHA act as hypolipidaemics, exert prophylactic effects on cardiovascular disease and may improve insulin sensitivity (references, see $[4,9])$. Therefore, current dietary recommendations favour a substantial decrease in the $n-6: n-3$ PUFA ratio $[6,9]$.

The effect of $n$-3 PUFA on fat deposition does not result from a reduction in food intake $[2-6,8,11]$, but rather reflects metabolic changes in several tissues $[6,12]$, such as stimulation of lipid oxidation and inhibition of lipogenesis in liver (references, see $[6,12]$ ), stimulation of fatty acid oxidation in muscle [13], as well as adaptive thermogenesis mediated by mitochondrial uncoupling protein 1 (UCP1) in brown fat $[2,14]$. In humans, whole-body lipid oxidation was increased by dietary fish oil [11]. The anti-adipogenic effect of $n-3$ PUFA may be also mediated by changes occurring in white adipose tissue itself $[5,15]$. Studies in rodents indicate a complex modulation of gene expression in white adipose tissue by $n-3$ PUFA $[6,12]$, especially in fat depots in the abdominal region, which are preferentially reduced $[3,5,6,8,16]$. The changes in gene expression suggest a decrease in lipogenesis and fatty acid release from adipocytes [5] and an enhancement of mitochondrial respiration [3] and glucose uptake [8] in these cells.

The main goals of this study were to investigate the effects of EPA and DHA, compared with ALA, on gene expression in white adipose tissue, and to test the hypothesis that these effects limit the development of obesity. Experiments were conducted in mice fed two types of highfat diet, either a semisynthetic diet (sHFf) or a composite diet based on chow (cHF; see Materials and methods and
[8]). The sHFf diet is well defined and made it possible to study the separate effects of EPA and DHA, and ALA, respectively. Similar purified diets have been used in previous studies in rodents. While these diets do not promote obesity in mice $[2-5,8,13,14]$, the cHF diet does $[8,17]$. We show here that replacement of some of the dietary lipids with EPA and DHA induced expression of mitochondrial genes in white adipose tissue, as well as the gene encoding their upstream regulatory factor, PPAR $\gamma$ coactivator-1 $\alpha$ (PPARGC1A, also known as PGC1 $\alpha$ ). This factor links nuclear receptors to the transcriptional program of mitochondrial biogenesis and oxidative metabolism in adipocytes and muscle cells [18-20] and to gluconeogenesis in liver [21]. We also demonstrate that the expression of the gene for carnitine palmitoyltransferase $1 \mathrm{~A}(\mathrm{Cptla})$ and oxidation of fatty acids was stimulated in epididymal but not subcutaneous white adipose tissue. Our data suggest that upregulation of mitochondrial biogenesis and induction of $\beta$-oxidation, as well as suppression of lipogenesis in adipocytes, are involved in the preferential reduction of lipid accumulation by EPA and DHA in abdominal fat.

\section{Materials and methods}

Animals and diet

Male C57BL/6J mice were imported from the Jackson Laboratory (Bar Habor, ME, USA) and bred at the Institute of Physiology for up to 16 generations. Male mice were used for the experiments. Animals were housed in a controlled environment $\left(20^{\circ} \mathrm{C} ; 12\right.$-h light-12-h dark cycle; light from $06.00 \mathrm{~h}$ ) with free access to water and standard chow [17]. At 4 months of age, singly caged animals were randomly assigned for 4 weeks to one of the high-fat diets described previously (see Tables 1, 2, 3 of Ref. [8] and the Electronic Supplementary Material [ESM] Tables 1 and 2); the sHFf diet, which contained $20 \%$ (wt/wt) flax-seed oil (rich in ALA) as the only lipid; or the sHFf-F2 diet, which had the same composition as the preceding diet except that $44 \%$ of lipids were replaced by $n-3$ PUFA concentrate containing 6\% EPA and 51\% DHA (EPAX 1050TG; Pronova Biocare, Lysaker, Norway; EPA/DHA concentrate). Some 4-month-old animals were also habituated for 2 weeks to the cHF diet, derived from standard chow and containing 35\% (wt/wt) lipids of very low $n$-3 PUFA content. These were then assigned for 5 weeks to the cHF diet or to the cHF-F1 diet, which had the same composition as the cHF diet except that $15 \%$ (wt/wt) of lipids were replaced with EPAX 1050TG [8]. Individual food intake was determined as before [8]. Mice were killed by cervical dislocation and subcutaneous dorsolumbar and epididymal white adipose tissue depots [8] were dissected. Tissues were either used immediately for measurement of fatty acid metabolism, or stored in liquid nitrogen for RNA analysis and immunoblotting. The experiments were conducted under the guidelines for the use and care of laboratory animals of the Institute of Physiology, Academy of Sciences of the Czech Republic. 
Table 1 Effects of EPA/DHA concentrate on body weight, fat depots and food consumption

\begin{tabular}{lcccc}
\hline & Diet & & & \\
\cline { 2 - 4 } & sHFf & sHFf-F2 & cHF & cHF-F1 \\
\hline Body weight (g) $^{\text {Initial }}$ & & & $27.5 \pm 0.6$ & $27.8 \pm 0.7$ \\
Final $^{\mathrm{a}}$ & $26.4 \pm 0.5$ & $26.4 \pm 0.4$ & $35.4 \pm 1.1$ & $33.4 \pm 0.7$ \\
Change $^{\mathrm{a}}$ & $26.9 \pm 0.7$ & $23.4 \pm 0.6^{\mathrm{b}}$ & $7.75 \pm 0.7$ & $6.25 \pm 0.6^{\mathrm{c}}$ \\
Epididymal fat weight (mg) $_{\text {Dorsolumbar fat weight (mg) }}$ & $0.4 \pm 0.5$ & $-2.9 \pm 0.8^{\mathrm{b}}$ & $2093 \pm 97$ & $1741 \pm 122^{\mathrm{c}}$ \\
Food consumption $^{\mathrm{a}}(\mathrm{kJ} /$ day per animal) & $481 \pm 38$ & $326 \pm 24^{\mathrm{b}}$ & $679 \pm 45$ & $640 \pm 40$ \\
$n$ & $215 \pm 12$ & $197 \pm 16$ & $65.5 \pm 3.1$ & $66.3 \pm 4.2$ \\
\hline
\end{tabular}

Data are means \pm SEM

Mice were fed semisynthetic diets (sHFf or sHFf-F2) and used for the analysis of gene expression in adipose tissue (Table 2, Fig. 2), or fed composite diets (cHF or cHF-F2) and used for the analysis of gene expression in collagenase-liberated adipocytes (Table 4)

${ }^{a}$ With respect to the whole period of dietary treatment

${ }^{\mathrm{b}}$ Statistically significant difference compared with sHFf diet

${ }^{\mathrm{c}}$ Statistically significant difference compared with cHF diet

\section{Differentiation of 3T3-L1 adipocytes}

Eleven days after induction of cell differentiation [22] and $24 \mathrm{~h}$ before RNA isolation, a complete change of the medium was performed using serum-free Dulbecco's modified Eagle medium containing $0.5 \%$ fatty acid-free BSA, $830 \mathrm{nmol} / \mathrm{l}$ insulin and $0.5 \mathrm{mmol} / \mathrm{l}$ oleic acid in the form of BSA/fatty acid sodium salt. Medium was further supplemented with ethanolic solution of fatty acid: (1) $0.2 \mathrm{mmol} / 1$ oleic acid; (2) $0.2 \mathrm{mmol} / 1 \mathrm{ALA}$; or (3) $0.2 \mathrm{mmol} / 1 \mathrm{DHA}$, and incubated for $1 \mathrm{~h}$ before use.

\section{RNA isolation}

Total RNA was isolated and purified using the TRIzol Reagent (Invitrogen, Carlsbad, CA, USA) according to the protocol of the supplier with additional phenol/chloroform extraction.

DNA and protein content

DNA was measured fluorometrically in tissue samples digested with proteinase $\mathrm{K}$, and protein concentration was assessed using the bicinchoninic acid procedure and BSA as standard [23].

\section{cDNA PCR subtraction}

RNA was isolated from epididymal fat pads pooled from six mice fed the sHFf or the sHFf-F2 diet. cDNA was generated using the SMART PCR cDNA Synthesis Kit (BD Biosciences, San Jose, CA, USA). To detect the genes upregulated by the sHFf-F2 diet, PCR-based cDNA subtraction was carried out with the PCR-Select cDNA Subtraction
Kit (BD Biosciences). Subtracted cDNA libraries were subcloned (T/A cloning) into bacteria and analysed by colony array hybridisation using ${ }^{32} \mathrm{P}$-labelled cDNA probes (DECAprime II DNA Labeling Kit; Ambion, Austin, TX, USA). Selected cDNAs were sequenced (DNA sequencer CEQ2000XL; Beckman Coulter, Harbor Boulevard, CA, USA) and DNA sequences were analysed using BLAST (GenBank, NIH).

Oligonucleotide microarray

Each RNA sample isolated from dorsolumbar and epididymal fat of sHFf and sHFf-F2 mice (nine animals per group) was indirectly labelled [24] and processed separately. In the reverse transcription step, aminoallyl dUTP was incorporated and then chemically coupled to fluorophores. RNA samples $(30 \mu \mathrm{g})$ were separately labelled with Cy5 dye. A common reference sample (a pool representing all the investigated samples) was also labelled with Cy3. Highdensity microarrays, based on commercial oligonucleotide library (Mouse 10k A set; MWG Biotech, Ebersberg bei München, Germany) were printed on epoxy slides (Quantifoil, Jena, Germany) using a MicroGrid II (Genomic Solutions, Ann Arbor, MI, USA), pretreated, hybridised overnight at $42^{\circ} \mathrm{C}$ in a humid hybridisation chamber, and read using a laser scanner (ScanArray Express; PerkinElmer, Boston, MA, USA). To correct for fluctuations in RNA sample size and the amount of DNA spotted, signals were normalised [25]. All Cy3 and Cy5 signals lower than 2.5 -fold background value were excluded from the analysis (approximately $60 \%$ of spots). Identification of differentially expressed genes was done using discriminant analysis, which is a supervised form of principal components analysis (Genemath software; Applied Maths, Sint-Martens-Lathem, Belgium). Samples were first assigned to their diet intervention group, then separated along the dimension of maximum 


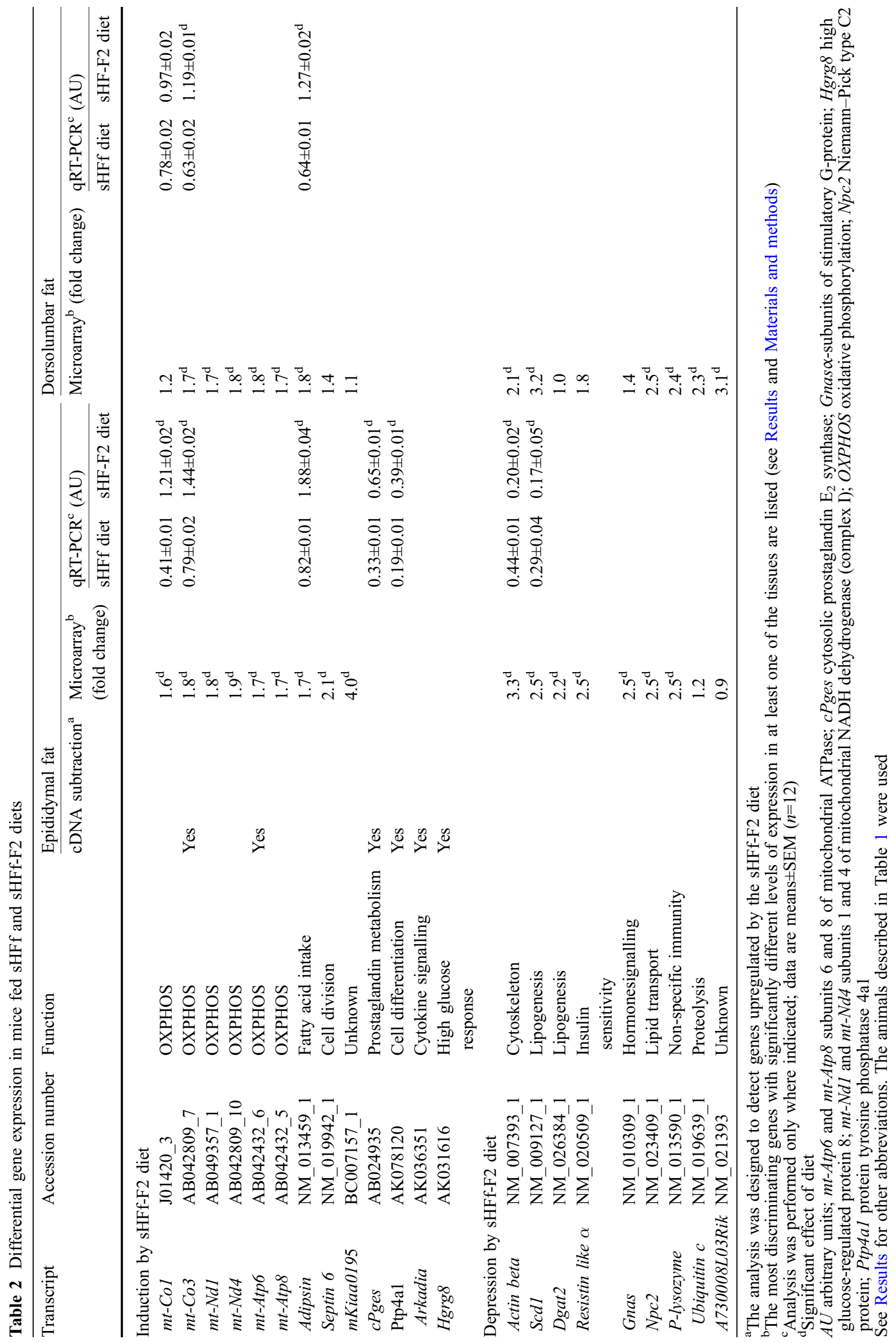


Table 3 Quantification of the protein components of the oxidative phosphorylation system in sHFf and sHFf-F2 diet-fed mice

\begin{tabular}{llllll}
\hline Fat depot & Diet & SDHA & MT-CO1 & COX6 & ATP5A1 \\
\hline Epididymal fat & sHFf & $0.25 \pm 0.04$ & $0.37 \pm 0.07$ & $0.28 \pm 0.02$ & $0.30 \pm 0.06$ \\
& sHFf-F2 & $0.48 \pm 0.06^{\mathrm{a}}$ & $0.68 \pm 0.08^{\mathrm{a}}$ & $0.67 \pm 0.14^{\mathrm{a}}$ & $0.44 \pm 0.03^{\mathrm{a}}$ \\
Dorsolumbar fat & sHFf & $0.42 \pm 0.04^{\mathrm{b}}$ & $0.34 \pm 0.06$ & $0.43 \pm 0.08$ & $0.33 \pm 0.07$ \\
& sHFf-F2 & $0.46 \pm 0.07$ & $0.32 \pm 0.07^{\mathrm{b}}$ & $0.38 \pm 0.07$ & $0.39 \pm 0.07$ \\
\hline
\end{tabular}

Individual antigens were quantified in crude cell membranes isolated from adipose tissues using immunoblots and their content was expressed in AU using isolated mouse liver mitochondria as a standard (see Fig. 1)

Data are means \pm SEM $(n=9)$. The effect of diet in fat depots (two-way ANOVA) was different, except for ATP5A1

${ }^{\text {a }}$ Significant differences between diets

${ }^{\mathrm{b}}$ Significant differences between fat depots

variation in gene expression between the groups and the $0.5 \%$ of genes that contributed most to the variation (the discriminating genes) were subsequently selected. Finally, from the selected genes only those genes were considered that exerted significantly different ( $t$-test) levels of expression in at least one of the tissues analysed.

\section{Quantitative real-time RT-PCR}

This analysis (qRT-PCR) was performed using a PCR kit (QuantiTect SYBR Green; Qiagen, Hilden, Germany) and LightCycler (Hoffman-La Roche, Basel, Switzerland). All samples were treated with DNAase. Dilution of cDNA samples before PCR quantification of mitochondrially encoded genes was 100-fold higher compared with nuclearencoded genes. Levels of all transcripts were correlated with that of the gene for cyclophilin- $\beta$ since quantity of this transcript is not affected by the dietary treatments [8] and is expressed in arbitrary units (AU). Lasergene software (DNAStar, Madison, WI, USA) was used to design oligonucleotide primers (see ESM Table 3).

\section{Quantification of mitochondrial proteins}

Frozen tissues were immersed in a buffer containing $0.25 \mathrm{~mol} / \mathrm{l}$ sucrose, $50 \mathrm{mmol} / \mathrm{l}$ Tris and $5 \mathrm{mmol} / \mathrm{l}$ EDTA (pH 7.5), and homogenised for $7 \mathrm{~min}$ on ice using a Teflon/glass homogeniser. Tissue fragments were removed by centrifugation for $10 \mathrm{~min}$ at $4^{\circ} \mathrm{C}$ and $600 \mathrm{~g}$ and crude cell membranes were separated from the supernatant by centrifugation for $35 \mathrm{~min}$ at $4^{\circ} \mathrm{C}$ and $100,000 \mathrm{~g}$. Samples of the crude cell membranes were analysed using SDS-PAGE and immunoblots [26]. Polyclonal rabbit antibody [26] was used $(1: 5,000)$ to detect cytochrome $c$ oxidase subunits I and VI (MT-CO1 and COX6). Monoclonal antibody was used to detect the $70 \mathrm{kDa}$ subunit of complex II (SDHA; 1:2,000; Molecular Probes A-11142, Eugene, OR, USA) and subunit $\alpha$ of $\mathrm{F}_{1}$-ATPase (ATP5A1; 1:200,000, lot 20D6 [27]). Peroxidase-labelled goat anti-mouse $\operatorname{IgG}(1: 1,000$, A8924; Sigma, St Louis, MO, USA) or goat anti-rabbit IgG $(1: 1,000$, F0382; Sigma) was used as a secondary antibody. Antigens were visualised by luminescence and detected on a LAS 1000 CCD camera system (Fuji, Tokyo, Japan). Liver mitochondria isolated from chow diet-fed mice were used as standard to quantify the relative content of each antigen.

Oxidation and synthesis of fatty acid in fragments of adipose tissue

Fatty acid oxidation was measured as the amount of radioactivity released into the medium from fragments $(50 \mathrm{mg}$ tissue chopped into about ten pieces using a razor blade) prelabelled with $\left[9,10(n)-{ }^{3} \mathrm{H}\right]$ oleic acid [28]. Synthesis of fatty acids was measured as before [22] except that the medium also contained $1 \mathrm{mM}$ pyruvate and ${ }^{3} \mathrm{H}_{2} \mathrm{O}(18.5 \mathrm{MBq} /$ $\mathrm{ml}$ ) was used instead of radioactive glucose.

\section{Statistics}

The data were evaluated by one-way (diet) or two-way ANOVA with one grouping factor (diet) and one trial factor (depot). Logarithmic or square root transformation was used to stabilise variance in cells when necessary. The Spearman correlation coefficient was used to evaluate the relationship between transcript levels. The level of significance for all tests was set at $p=0.05$.

\section{Results}

Screening for genes regulated by EPA and DHA in white adipose tissue

To identify the genes specifically regulated in white adipose tissue by marine-derived $n$-3 PUFA, mice were maintained for 4 weeks on the sHFf diet, rich in ALA and free of DHA or EPA, while the other mice were fed a similar diet, except that $44 \%$ of dietary lipids were replaced by EPA/DHA concentrate (sHFf-F2 diet). In accordance with previous results [8], animals fed the sHFf diet maintained stable body weight during 4 weeks of the treatment, while in the sHFf-F2 group the mean body weight declined by $11 \pm 2 \%$. The weight of epididymal fat was $30 \pm 3 \%$ lower than in the sHFf group (mean \pm SEM of four independent experiments, $n=7-12$ ), while the weight of dorsolumbar fat did not change significantly. As observed before [8], food consumption was not affected (Table 1). 
First, cDNA PCR subtraction was used to identify genes upregulated in the sHFf-F2-fed mice, starting from total RNA prepared from epididymal pads pooled from mice fed either the sHFf or the sHFf-F2 diet. In total, 52 clones were identified corresponding to 19 differentially expressed genes, six of them having known functions (Table 2): genes for cytochrome $c$ oxidase subunit III (mt-Co3) and subunit 6 of ATPase (mt-Atp6), i.e. the key components of the mitochondrial oxidative phosphorylation system, encoded by mitochondrial DNA, and four nuclear genes engaged in regulatory pathways.

Secondly, oligonucleotide microarray analysis was applied to RNA samples isolated from both epididymal and dorsolumbar fat. Tissues from individual animals fed either sHFf or sHFf-F2 diet (Table 1) were processed and analysed separately. Only $0.5 \%$ of the genes most discriminating in both directions (see Materials and methods) between the sHFf and sHFf-F2 diets were considered (Table 2). In accordance with the results of cDNA PCR subtraction, the majority of the most upregulated genes in both fat depots of the sHFf-F2-fed mice belong to the mitochondrial genome and code for components of the oxidative phosphorylation system (complex I, cytochrome $c$ oxidase and complex V, ATP synthase). Also, the nuclear gene for adipsin, the adipocyte hormone engaged in the control of fatty acid intake [29], was upregulated. Conversely, the most downregulated genes in the sHFf-F2-fed animals were those supporting lipogenesis and encoding stearoyl-CoA desaturase ( $S c d 1$ [30]), in both epididymal and subcutaneous fat depots, and acyl-CoA:diacylglycerol acyltransferase (Dgat2), in the former depot only (Table 2).

Thirdly, seven of the 18 differentially expressed genes were verified using qRT-PCR (Table 1). The differences in the expression of the gene for $m t-C o l$ were more pronounced in the epididymal than in the dorsolumbar fat depot (Table 2).

Specific induction of mitochondrial proteins in epididymal fat by the sHFf-F2 diet

Subunits of the mitochondrial oxidative phosphorylation complexes were quantified in crude cell membranes prepared from white adipose tissue using immunoblots (Fig. 1, Table 3). In epididymal fat, levels of SDHA, MT-CO1, COX6 and ATP5A1 were elevated approximately 2-fold by the sHFf-F2 diet compared with the sHFf diet, resulting, in the case of MT-CO1, in higher levels in epididymal than in dorsolumbar fat in the sHFf-F2-fed animals. In the dorsolumbar fat, no effect of the diet on any of the analysed antigens was observed. In contrast to the differential effect of the sHF-F2 diet on the quantity of SDHA and ATP5A1 in two fat depots, qRT-PCR analysis $(n=7-9)$ revealed significant induction of transcripts for these genes by sHF-F2 diet in both epididymal fat (Sdha, $1.35 \pm 0.16$ vs $2.71 \pm 0.35$ AU; Atp5a1, $1.82 \pm 0.08$ vs $3.97 \pm 0.75$ AU) and subcutaneous fat (Sdha, $1.96 \pm 0.19$ vs $2.75 \pm 0.26$ AU; Atp $5 a 1,1.44 \pm$ 0.23 vs $3.31 \pm 0.54 \mathrm{AU})$.



Protein

subunit

SDHA

MT-CO1

cox6

ATP5A1

Fig. 1 Immunoblotting of mitochondrial proteins in epididymal fat of the mice fed sHFf and mice fed sHFf-F2 diets. The analysis was performed using crude cell membranes isolated from adipose tissue. The amount of protein in each lane is indicated. Isolated mouse liver mitochondria were used as a standard (see Table 3)

Induction of Ppargcla, Nrf1, Cpt1a, fatty acid oxidation and depression of fatty acid synthesis in white adipose tissue by the sHFf-F2 diet

We hypothesised that the induction of PPARGC1A, as well as NRF1 [18, 19], which is a downstream target for PPARGC1A and orchestrates mitochondrial biogenesis [19], is involved in the effect of EPA/DHA concentrate on white adipose tissue. qRT-PCR analysis revealed approximately 3.4-fold induction of both Ppargcla and Nrfl transcripts in epididymal fat by the sHFf-F2 diet compared with the sHFf diet (Figs. 2a and b). Both genes tended to be upregulated also in dorsolumbar fat, but the effects were much smaller and not statistically significant. Also, CPT1, which is necessary for the translocation of fatty acid into mitochondria, represents a downstream target for PPARG C1A [31]. qRT-PCR analysis revealed that the liver isoform of the gene encoding CPT1 (Cptla) was upregulated by the sHFf-F2 diet in epididymal but not in dorsolumbar fat (Fig. 2c). Positive correlations between the expressions of genes encoding PPARGC1A and CPT1A within identical tissues and diets were observed, while correlations between the expression of genes encoding PPARGC1A and NRF1 were not statistically significant (Fig. $2 \mathrm{~d}$ and legend). The upregulation of the gene for CPT1A suggests stimulation of fatty acid oxidation in the epididymal fat of the sHFf-F2 mice. Indeed, oxidation of oleate was 1.5- to 1.8 -fold higher in epididymal fat of the sHFf-F2 compared with the sHFf mice, when the rate of oxidation was related either to tissue weight, protein, or DNA (Fig. 3). In dorsolumbar fat, similar rates were observed in both diet groups (Fig. 3). Fatty acid synthesis was lower in epididymal fat of sHFfF2 than sHFf mice $\left(83 \pm 7\right.$ vs $114 \pm 12 \mathrm{dpm}$ of ${ }^{3} \mathrm{H}_{2} \mathrm{O}$ incorporated into saponifiable fatty acids per $\mathrm{mg}$ of tissue DNA; $n=7$ ).

Levels of acyl-CoA oxidase 1 (Acoxl) mRNA, the marker of peroxisomal fatty acid oxidation, were significantly higher in mice fed the sHFf-F2 diet than in those fed the sHFf diet, in both epididymal $(0.27 \pm 0.05$ vs $0.14 \pm 0.02 \mathrm{AU})$ 
a

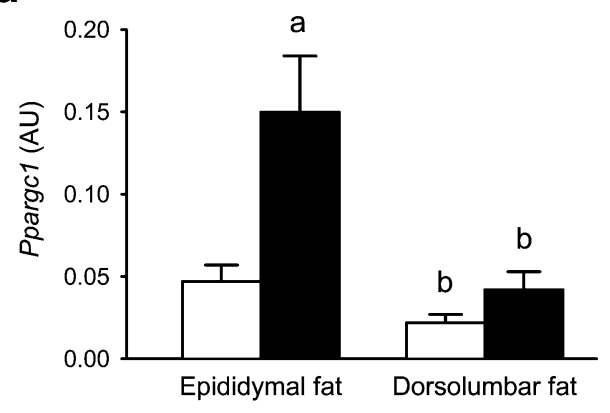

C

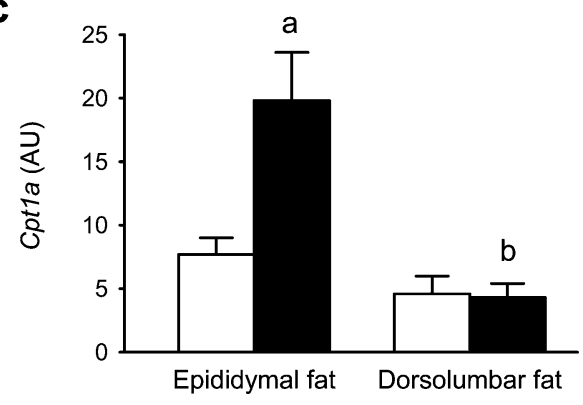

Fig. 2 Gene expression in adipose tissue. a-c Using qRT-PCR, transcript levels were evaluated in total RNA isolated from adipose tissue depots of mice fed the sHFf diet (white bars) or the sHFf-F2 diet (black bars). For all transcripts, the effect of diet in fat depots was different (two-way ANOVA). Significant differences between diets; significant differences between fat depots. Data are means \pm SEM $(n=9-15)$. d Correlation between Ppargcla and Cptla mRNA

and dorsolumbar $(0.09 \pm 0.02$ vs $0.04 \pm 0.01$ AU) adipose tissue. Thus, in contrast to the gene encoding CPT1A, similar stimulation of transcription of the gene for ACOX1 was observed in both fat depots of sHFf-F2 mice $(n=13)$.

In contrast to white adipose tissue, especially the epididymal fat, no significant changes in the expression of Ppargcla, Nrfl and Cptla were observed in liver (not shown). Only the levels of Acox 1 mRNA were significantly higher in mice fed the sHFf-F2 than in those fed the sHFf $\operatorname{diet}(32 \pm 1.5$ vs $21 \pm 1.3 \mathrm{AU} ; n=5)$. b



d

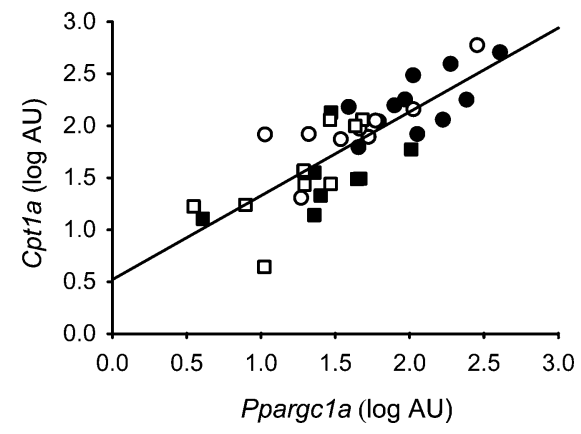

levels in epididymal fat of the mice fed sHFf (white circles) and sHFf-F2 (black circles) and in dorsolumbar fat of the mice fed sHFf (white squares) and sHFf-F2 (black squares). Spearman correlation coefficients for each set of data were $0.72-0.83$. Corresponding values for the correlation between Ppargcla and $\mathrm{Nrfl}$ transcripts were 0.38-0.62. Only the correlations between Ppargcla and Cptla transcripts $(\mathbf{d})$ were statistically significant $(p<0.01)$

Upregulation of the markers of mitochondrial biogenesis and $\beta$-oxidation and suppression of lipogenic genes in adipocytes from mice fed the composite diet cHF-F1

These experiments were designed to verify whether mitochondrial biogenesis and $\beta$-oxidation are stimulated when induction of obesity by the cHF diet is reduced by replacement of only $15 \%$ of dietary lipids by EPA/DHA concentrate (cHF-F1 diet). In accordance with our previous

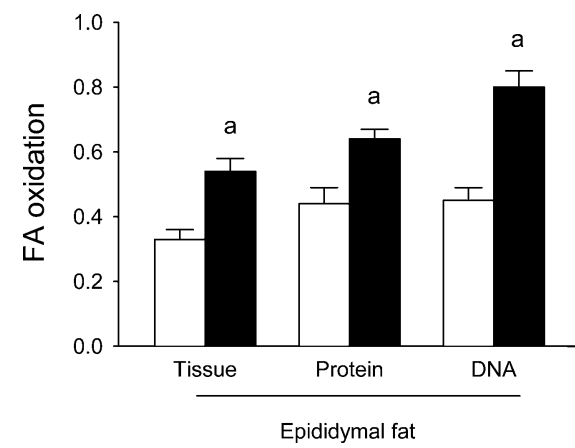

Fig. 3 Oxidation of oleate in fat depots of mice fed sHFf (white bars) or sHFf-F2 (black bars). Activity is expressed as pmol fatty acids $\cdot \mathrm{h}^{-1} \cdot \mathrm{mg}^{-1}$ tissue (Tissue), pmol fatty acids $\cdot \mathrm{h}^{-1} \cdot \mathrm{mg}^{-1}$ protein $\cdot 10^{-2}$ (Protein), or pmol fatty acids $\cdot \mathrm{h}^{-1} \cdot \mu^{-1}$ DNA $(D N A)$. There was a different effect of diet in fat depots (two-way ANOVA). Data are means \pm SEM $(n=9)$. The tissue
DNA concentration was $0.78 \pm 0.08$ and $0.76 \pm 0.11 \mu \mathrm{g}$ DNA $/ \mathrm{mg}$ tissue in epididymal fat of sHFf and sHFf-F2 mice, respectively. Corresponding values for dorsolumbar fat were $1.27 \pm 0.16$ and $1.05 \pm 0.20$. Significant difference between diets 
Table 4 Gene expression in adipocytes isolated from epididymal fat of mice fed cHF or cHF-F1 diet

\begin{tabular}{lllllll}
\hline Diet & Ppargc1a & mt-Co3 & Cpt1a & Scd1 & Ucp2 & Ucp3 \\
\hline cHF & $0.14 \pm 0.03$ & $9.25 \pm 1.02$ & $1.40 \pm 0.32$ & $1.57 \pm 0.18$ & $0.87 \pm 0.11$ & $0.82 \pm 0.02$ \\
cHF-F1 & $0.51 \pm 0.09^{\mathrm{a}}$ & $12.81 \pm 0.64^{\mathrm{a}}$ & $2.32 \pm 0.61^{\mathrm{a}}$ & $0.40 \pm 0.08^{\mathrm{a}}$ & $0.40 \pm 0.09^{\mathrm{a}}$ & $1.01 \pm 0.03$ \\
\hline
\end{tabular}

Data are means $\pm \operatorname{SEM}(n=7)$

${ }^{\text {a }}$ Significant difference between diets

study [8], a significant $22 \pm 7 \%$ reduction of body weight gain was observed in the cHF-F1-fed mice compared with the cHF-fed mice after 5 weeks of the treatment. The weight of epididymal fat in the cHF-F1-fed mice was $24 \pm 3 \%$ lower than in the cHF group (mean \pm SEM of three independent experiments, $n=10-13$ ), while the weight of dorsolumbar fat was not significantly different. As observed before [8], food consumption was not affected (see Table 1 for data from a typical experiment).

In animals fed the cHF and cHF-F1 diets (Table 1), gene expression was studied using RNA prepared from the adipocytes isolated from epididymal fat (Table 4). Expression of the genes for PPARGC1A, MT-CO3 and CPT1A was enhanced by the cHF-F1 diet compared with the cHF diet, while the gene encoding SCD1 was down-regulated. Since PPARGC1A can induce the expression of the gene for mitochondrial UCP1 $[18,20]$ and marine-derived $n-3$ PUFA have been shown to induce expression of this gene in brown fat [14], the Ucpl transcript was also measured. However, it was barely detectable (levels were at least four orders of magnitude lower than in interscapular brown fat) and no induction of the Ucp 1 transcript by cHF-F1 diet was observed (not shown). Levels of Ucp2 transcript were lower in adipocytes from the cHF-F1-fed than the cHF-fed mice, while levels of $U c p 3$ transcript were similar in the two groups of mice (Table 4).

\section{Direct effect of $n$-3 PUFA on adipocytes}

3T3-L1 adipocytes differentiated in cell culture were incubated for $24 \mathrm{~h}$ with various fatty acids: oleate alone, oleate plus ALA, or oleate plus DHA, and Ppargcla and Nrfl transcripts were quantified (Fig. 4). Compared with oleate, both ALA and DHA significantly increased the levels of both transcripts. The stimulation by DHA seemed to be more pronounced than that by ALA, but the dif- ference was not statistically significant. Similar results were obtained in primary cultures of adipocytes (not shown).

\section{Discussion}

The results of this study demonstrate the involvement of white adipose tissue, in addition to the liver, brown fat and other organs, in the mechanism whereby marine-derived $n$-3 PUFA reduce adiposity and body weight. Our finding that EPA/DHA concentrate can specifically stimulate expression of Ppargcla and Cpt1a, increase the content of mitochondrial proteins and induce $\beta$-oxidation in epididymal but not dorsolumbar fat is consistent with the preferential reduction of adipose tissue accumulation in the abdomen.

The idea that changes induced in white adipocytes by EPA and DHA could contribute to the reduction of obesity is strongly supported by the upregulation of genes encoding PPARGC1A and MT-CO3 and downregulation of the gene encoding SCD1 in adipocytes prepared from epididymal fat of the cHF-F1-fed mice. Under these conditions (mouse fed the cHF-F1 diet), only $15 \%$ of dietary fat was replaced by EPA/DHA concentrate, resulting in a substitution of about $10 \%$ of the fat for EPA/DHA. This led to a significant reduction in weight gain compared with mice fed the cHF diet.

Increased expression of $\mathrm{Nrfl}$ is consistent with the upregulation of genes for mitochondrial proteins by EPA/ DHA. The majority of these genes (i.e. $m t$-Col, $m t-C o 3, m t-$ $N d 1, m t-N d 4, m t-A t p 6$ and $m t-A t p 8)$ are encoded by mitochondrial DNA. However, immunoblotting analysis revealed that the biosynthesis of nuclear-encoded mitochondrial proteins (COX6, SDHA and ATP5A1) is also upregulated. Expression of genes encoding PPARGC1A and NRF1 is more stimulated in epididymal than in dorsolumbar
Fig. 4 Effect of n-3 PUFA on gene expression in 3T3-L1 cells. Postconfluent cells were treated for $24 \mathrm{~h}$ with oleate, oleate plus ALA, or oleate plus DHA (see Materials and methods), and transcripts were quantified using qRT-PCR. Data are means \pm SEM $(n=12)$. Significant differences versus oleate a

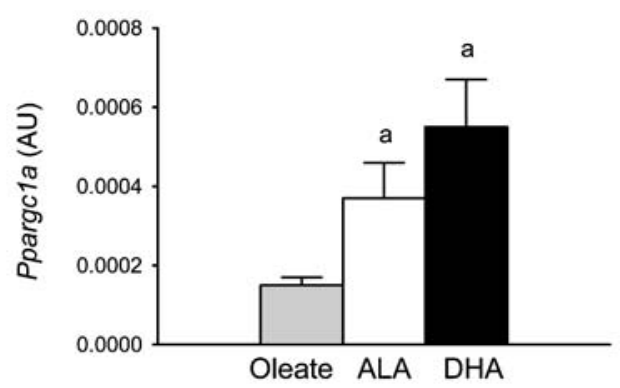

b

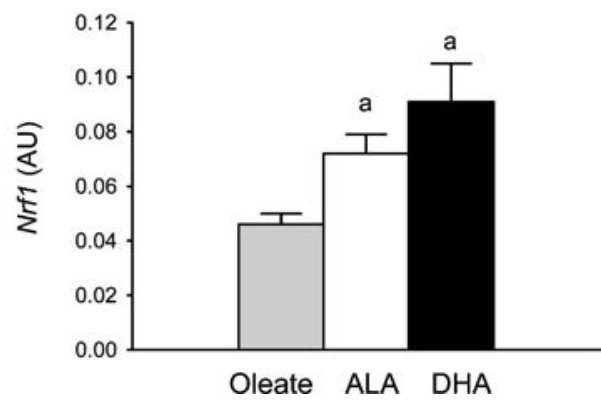


fat, and striking fat depot-specific differences were observed in the effect of EPA/DHA on the expression of the gene encoding CPT1A, fatty acid oxidation activity and the quantity of oxidative phosphorylation proteins. In contrast, EPA/DHA induced transcripts for most of the mitochondrial proteins as well as the marker of peroxisomal fatty acid oxidation, Acoxl, to a similar extent in both fat depots. These results suggest distinct post-transcriptional control in different fat depots of the expression of the genes for mitochondrial proteins and the involvement of mitochondria rather than peroxisomes in the stimulatory effect of EPA and DHA on fatty acid oxidation in epididymal fat.

Our in vitro experiments suggest that $n$-3 PUFA can modulate gene expression in white adipose tissue by direct interaction with adipocytes. PUFA of the $n-3$ series and their metabolites, eicosanoids [6], are ligands [32] of various isoforms of the peroxisome proliferator-activated receptor (PPAR [33-35]). All PPARs ( $\alpha, \gamma$ and $\delta$ ) physically interact with the gene encoding PPARGC1A [18, 34, 35], which is upregulated by EPA/DHA in adipocytes. A recent study indicates that treatment of $o b / o b$ mice with rosiglitazone, a PPAR $\gamma$ ligand of the thiazolidinedione family and a widely used antidiabetic drug, increases mitochondrial mass, palmitate oxidation and the expression of genes encoding PPARGC1A, CPT1A and UCP1 in epididymal fat [36]. Except for the upregulation of the gene for UCP1, all these effects are similar to those elicited in white adipose tissue by EPA/DHA, suggesting that both $n$-3 PUFA and rosiglitazone induce similar metabolic changes in white adipose tissue cells, while increasing mitochondrial oxidative capacity and stimulating glucose uptake into adipocytes. However, in contrast to EPA/DHA, thiazolidinediones also support an adipogenic programme [36]. Surprisingly, rosiglitazone induces the accumulation of $n$-3 PUFA in white adipose tissue of diabetic mice [37]. Whether the mechanism of the effect of rosiglitazone depends on the accumulation of $n-3$ PUFA in adipocytes remains to be clarified.

Forced expression of the gene for PPARGC1A in both 3T3-F442A (references, see [18]) and human adipocytes [20] induces the conversion of adipocytes to energy-dissipating cells, including the recruitment of the gene for UCP1. Low levels of transgenic UCP1 in white adipose tissue may protect mice against obesity [17] while stimulating oxidation of fatty acids [28] and mitochondrial biogenesis [23], and depressing lipogenesis [22] in adipocytes. Also, leptin $[38,39], \beta_{3}$-adrenergic agonists [40] and bezafibrate [41] have similar effects in rodents [38,41], including induction of the genes for CPT1A $[39,41]$ and PPARGC1A [38] in white adipose tissue. Apparently, various stimuli can convert adipocytes into fat-burning cells and depress the accumulation of body fat, even without induction of UCPs in these cells, as documented by the effect of EPA/DHA. Lack of recruitment of UCP1 in the EPA/DHA-treated mice may be explained by the absence of other cofactors or hormones required in addition to PPARGC1A $[18,20]$. The absence of UCP1 also suggests that the increase in fatty acid oxidation occurs in white rather than brown fat cells. This idea is supported by the induction of the gene for
CPT1A, which is preferentially expressed in white adipose tissue in mice [42]. Because of the low activity of CPT1A, the rate of $\beta$-oxidation in white adipose tissue is normally low and fatty acids are directed to esterification [42, 43]. Thus, the enhancement of fatty acid oxidation in white adipose tissue by EPA/DHA concentrate could depend on the increase in the expression of the gene encoding CPT1A. Decreased production of malonyl CoA due to suppression of lipogenesis may result in further augmentation of fatty acid oxidation in mitochondria via the CPT1A-mediated mechanism [44].

It was found recently that expression of the gene encoding PPARGC1A is reduced in the adipose tissue of morbidly obese subjects [45] and inversely correlated with adipose cell mass [46], and that Ppargcla polymorphisms segregate with obesity [47] and type 2 diabetes [48]. Onset of obesity in $o b / o b$ mice is associated with downregulation of genes coding for mitochondrial proteins [36], and heat production by white adipose tissue cells from obese humans is lower than in lean individuals [49]. All these findings are compatible with the hypothesis that changes in fuel partitioning and reduced oxidation of lipids in white adipocytes contribute to the development of obesity and type 2 diabetes.

In conclusion, we show for the first time that dietary EPA and DHA preferentially upregulate genes for mitochondrial proteins, including their regulatory genes Ppargcla and $N r f 1$, and increase $\beta$-oxidation while depressing lipogenesis in abdominal fat. This metabolic switch is induced independently of ALA intake and could reduce the development of obesity. Relatively minor changes in the composition of lipids in an obesity-promoting diet exert surprisingly pronounced effects. Our findings have important implications for the design of novel dietary and pharmacological strategies for the prevention and treatment of obesity and the metabolic syndrome.

Acknowledgements We thank J. Bemova, S. Hornova, D. Salkova, V. Fialova, C. Vink for technical assistance, J. Vorlicek for statistical analysis, and Drs A. Kotyk and V. Mohamed-Ali for critical reading of the manuscript. This work was supported by the Grant Agency of the ASCR (KJB5011410 and KJB5011303), the Czech Science Foundation (303/05/2580 and 303/03/0749), the MEYS (LL00A079), research projects AVOZ 50110509 and 1M6837805002 (Czech Republic), Pronova Biocare a.s. (Norway) and the Ministry of Agriculture, Nature Management and Food Control (The Netherlands).

\section{References}

1. Kopelman PG (2000) Obesity as a medical problem. Nature 404:635-643

2. Oudart H, Groscolas R, Calgari C et al (1997) Brown fat thermogenesis in rats fed high-fat diets enriched with $n-3$ polyunsaturated fatty acids. Int J Obes Relat Metab Disord 21:955-962

3. Hun CS, Hasegawa K, Kawabata T, Kato M, Shimokawa T, Kagawa Y (1999) Increased uncoupling protein 2 mRNA in white adipose tissue, and decrease in leptin, visceral fat, blood glucose, and cholesterol in KK-Ay mice fed with eicosapentaenoic and docosahexaenoic acids in addition to linolenic acid. Biochem Biophys Res Commun 259:85-90 
4. Ikemoto S, Takahashi M, Tsunoda N, Maruyama K, Itakura H, Ezaki O (1996) High-fat diet-induced hyperglycemia and obesity in mice: differential effects of dietary oils. Metabolism 45:1539-1546

5. Raclot T, Groscolas R, Langin D, Ferre P (1997) Site-specific regulation of gene expression by $n-3$ polyunsaturated fatty acids in rat white adipose tissues. J Lipid Res 38:1963-1972

6. Azain MJ (2004) Role of fatty acids in adipocyte growth and development. J Anim Sci 82:916-924

7. Hill JO, Peters JC, Lin D, Yakubu F, Greene H, Swift L (1993) Lipid accumulation and body fat distribution is influenced by type of dietary fat fed to rats. Int J Obes Relat Metab Disord 17:223-236

8. Ruzickova J, Rossmeisl M, Prazak T et al (2004) Omega-3 PUFA of marine origin limit diet-induced obesity in mice by reducing cellularity of adipose tissue. Lipids 39:1177-1185

9. Ruxton CH, Reed SC, Simpson MJ, Millington KJ (2004) The health benefits of omega-3 polyunsaturated fatty acids: a review of the evidence. J Hum Nutr Diet 17:449-459

10. Sinclair AJ, Attar-Bashi NM, Li D (2002) What is the role of alpha-linolenic acid for mammals? Lipids 37:1113-1123

11. Couet C, Delarue J, Ritz P, Antoine J-M, Lamisse F (1997) Effect of dietary fish oil on body fat mass and basal fat oxidation in healthy adults. Int J Obes Relat Metab Disord 21:637-643

12. Lapillonne A, Clarke SD, Heird WC (2004) Polyunsaturated fatty acids and gene expression. Curr Opin Clin Nutr Metab Care 7:151-156

13. Baillie RA, Takada R, Nakamura M, Clarke SD (1999) Coordinate induction of peroxisomal acyl-CoA oxidase and UCP-3 by dietary fish oil: a mechanism for decreased body fat deposition. Prostaglandins Leukot Essent Fat Acids 60:351356

14. Takahashi Y, Ide T (2000) Dietary $n-3$ fatty acids affect mRNA level of brown adipose tissue uncoupling protein 1 , and white adipose tissue leptin and glucose transporter 4 in the rat. Br J Nutr 84:175-184

15. Raclot T, Oudart H (1999) Selectivity of fatty acids on lipid metabolism and gene expression. Proc Nutr Soc 58:633-646

16. Newman RE, Bryden WL, Fleck E et al (2002) Dietary $n-3$ and $n-6$ fatty acids alter avian metabolism: metabolism and abdominal fat deposition. Br J Nutr 88:11-18

17. Kopecky J, Hodny Z, Rossmeisl M, Syrovy I, Kozak LP (1996) Reduction of dietary obesity in the aP2-Ucp transgenic mice: physiology and adipose tissue distribution. Am J Physiol 270: E768-E775

18. Puigserver P, Spiegelman BM (2003) Peroxisome proliferatoractivated receptor-gamma coactivator 1 alpha (PGC-1 alpha): transcriptional coactivator and metabolic regulator. Endocr Rev 24:78-90

19. Kelly DP, Scarpulla RC (2004) Transcriptional regulatory circuits controlling mitochondrial biogenesis and function. Genes Dev 18:357-368

20. Tiraby C, Tavernier G, Lefort C et al (2003) Acquirement of brown fat cell features by human white adipocytes. J Biol Chem 278:33370-33376

21. Lin J, Wu PH, Tarr PT et al (2004) Defects in adaptive energy metabolism with CNS-linked hyperactivity in PGC-1alpha null mice. Cell 119:121-135

22. Rossmeisl M, Syrový I, Baumruk F, Flachs P, Janovská P, Kopecký J (2000) Decreased fatty acid synthesis due to mitochondrial uncoupling in adipose tissue. FASEB J 14:17931800

23. Rossmeisl M, Barbatelli G, Flachs P et al (2002) Expression of the uncoupling protein 1 from the $a P 2$ gene promoter stimulates mitochondrial biogenesis in unilocular adipocytes in vivo. Eur J Biochem 269:1-10

24. Henegariu O, Bray-Ward P, Ward DC (2000) Custom fluorescent-nucleotide synthesis as an alternative method for nucleic acid labeling. Nat Biotechnol 18:345-348
25. Pellis L, Franssen-van Hal NL, Burema J, Keijer J (2003) The intraclass correlation coefficient applied for evaluation of data correction, labeling methods, and rectal biopsy sampling in DNA microarray experiments. Physiol Genomics 16:99-106

26. Antonicka H, Floryk D, Klement P et al (1999) Defective kinetics of cytochrome $c$ oxidase and alteration of mitochondrial membrane potential in fibroblasts and cytoplasmic hybrid cells with the mutation for myoclonus epilepsy with ragged-red fibres ('MERRF') at position 8344 nt. Biochem J 342:537-544

27. Moradi-Ameli M, Godinot C (1983) Characterization of monoclonal antibodies against mitochondrial F1-ATPase. Proc Natl Acad Sci U S A 80:6167-6171

28. Matejkova O, Mustard KJ, Sponarova J et al (2004) Possible involvement of AMP-activated protein kinase in obesity resistance induced by respiratory uncoupling in white fat. FEBS Lett 569:245-248

29. Cianflone K, Xia Z, Chen LY (2003) Critical review of acylationstimulating protein physiology in humans and rodents. Biochim Biophys Acta 1609:127-143

30. Dobrzyn A, Ntambi JM (2004) The role of stearoyl-CoA desaturase in body weight regulation. Trends Cardiovasc Med $14: 77-81$

31. Song S, Zhang Y, Ma K et al (2004) Peroxisomal proliferator activated receptor gamma coactivator (PGC-1alpha) stimulates carnitine palmitoyltransferase I (CPT-Ialpha) though the first intron. Biochim Biophys Acta 1679:164-173

32. Forman BM, Chen J, Evans RM (1997) Hypolipidemic drugs, polyunsaturated fatty acids, and eicosanoids are ligands for peroxisome proliferator-activated receptors alpha and delta. Proc Natl Acad Sci U S A 94:4312-4317

33. Puigserver $\mathrm{P}$, Wu Z, Park CW, Graves R, Wright $\mathrm{M}$, Spiegelman BM (1998) A cold-inducible coactivator of nuclear receptors linked to adaptive thermogenesis. Cell 92:829-839

34. Vega RB, Huss JM, Kelly DP (2000) The coactivator PGC-1 cooperates with peroxisome proliferator-activated receptor alpha in transcriptional control of nuclear genes encoding mitochondrial fatty acid oxidation enzymes. Mol Cell Biol 20:1868-1876

35. Wang YX, Lee CH, Tiep $\mathrm{S}$ et al (2003) Peroxisomeproliferator-activated receptor delta activates fat metabolism to prevent obesity. Cell 113:159-170

36. Wilson-Fritch L, Nicoloro S, Chouinard M et al (2004) Mitochondrial remodeling in adipose tissue associated with obesity and treatment with rosiglitazone. J Clin Invest 114: 1281-1289

37. Watkins SM, Reifsnyder PR, Pan HJ, German JB, Leiter EH (2002) Lipid metabolome-wide effects of the PPARgamma agonist rosiglitazone. J Lipid Res 43:1809-1817

38. Orci L, Cook WS, Ravazzola M et al (2004) Rapid transformation of white adipocytes into fat-oxidizing machines. Proc Natl Acad Sci U S A 101:2058-2063

39. Wang MY, Lee Y, Unger RH (1999) Novel form of lipolysis induced by leptin. J Biol Chem 274:17541-17544

40. Himms-Hagen J, Melnyk A, Zingaretti MC, Ceresi E, Barbatelli G, Cinti S (2000) Multilocular fat cells in WAT of CL-316243-treated rats derive directly from white adipocytes. Am J Physiol Cell Physiol 279:C670-C681

41. Cabrero A, Alegret M, Sanchez RM, Adzet T, Laguna JC, Vazquez M (2001) Bezafibrate reduces mRNA levels of adipocyte markers and increases fatty acid oxidation in primary culture of adipocytes. Diabetes 50:1883-1890

42. Brown NF, Hill JK, Esser V et al (1997) Mouse white adipocytes and 3T3-L1 cells display an anomalous pattern of carnitine palmitoyltransferase (CPT) I isoform expression during differentiation. Inter-tissue and inter-species expression of CPT I and CPT II enzymes. Biochem J 327:225-231

43. Martin BR, Denton RM (1970) The intracellular localization of enzymes in white-adipose-tissue fat-cells and permeability properties of fat-cell mitochondria. Biochem J 117:861-877 
44. Ruderman NB, Saha AK, Kraegen EW (2003) Minireview: malonyl CoA, AMP-activated protein kinase, and adiposity. Endocrinology 144:5166-5171

45. Semple RK, Crowley VC, Sewter CP et al (2004) Expression of the thermogenic nuclear hormone receptor coactivator PGC1alpha is reduced in the adipose tissue of morbidly obese subjects. Int J Obes Relat Metab Disord 28:176-179

46. Yang X, Enerback S, Smith U (2003) Reduced expression of FOXC2 and brown adipogenic genes in human subjects with insulin resistance. Obes Res 11:1182-1191
47. Esterbauer H, Oberkofler H, Linnemayr V et al (2002) Peroxisome proliferator-activated receptor-gamma coactivator1 gene locus: associations with obesity indices in middle-aged women. Diabetes 51:1281-1286

48. Oberkofler H, Linnemayr V, Weitgasser R et al (2004) Complex haplotypes of the PGC-1alpha gene are associated with carbohydrate metabolism and type 2 diabetes. Diabetes 53: 1385-1393

49. Bottcher H, Furst P (1997) Decreased white fat cell thermogenesis in obese individuals. Int J Obes 21:439-444 